\title{
On the Union of Arithmetic Progressions
}

\author{
Shoni Gilboa* $\quad$ Rom Pinchasi ${ }^{\dagger}$
}

October 16, 2018

\begin{abstract}
We show that for every $\varepsilon>0$ there is an absolute constant $c(\varepsilon)>0$ such that the following is true. The union of any $n$ arithmetic progressions, each of length $n$, with pairwise distinct differences must consist of at least $c(\varepsilon) n^{2-\varepsilon}$ elements. We observe, by construction, that one can find $n$ arithmetic progressions, each of length $n$, with pairwise distinct differences such that the cardinality of their union is $o\left(n^{2}\right)$. We refer also to the non-symmetric case of $n$ arithmetic progressions, each of length $\ell$, for various regimes of $n$ and $\ell$.
\end{abstract}

\section{Introduction}

For integers $n>1$ and $\ell>1$ let $u_{\ell}(n)$ be the minimum possible cardinality of a union of $n$ arithmetic progressions, each of length $\ell$, with pairwise distinct differences.

Clearly, $u_{\ell}(n) \leq n \ell$, but this inequality is not tight in general, not even up to a multiplicative absolute constant.

For small values of $\ell$ it is not hard to see, for instance, that $u_{2}(n)=\left\lceil\frac{1}{2}+\sqrt{2 n+\frac{1}{4}}\right\rceil$. Ruzsa proved (in [7]) that there is an absolute constant $c>0$, such that for any sufficiently large integer $m$ there is a set $A$ of $m$ integers such that $|A+A| \leq m^{2-c}$ but $|A-A| \geq m^{2}-m^{2-c}$. For any positive $d \in A-A$ choose $a_{d}, b_{d} \in A$ such that $b_{d}-a_{d}=d$. Now $\left(2 a_{d}, a_{d}+b_{d}, 2 b_{d}\right)_{d \in A-A}$ is a set of $\frac{|A-A|-1}{2}$ arithmetic progressions of length 3 with pairwise distinct differences, and their union is contained in $(A+A) \cup 2 A$. We thus obtain at least $\frac{m^{2}-m^{2-c}-1}{2}$ arithmetic progressions of length 3 , with pairwise distinct differences, such that the cardinality of their union is at most $m^{2-c}+m$. It follows that $u_{3}(n)=O\left(n^{1-\frac{c}{2}}\right)$.

The trivial upper bound $u_{\ell}(n) \leq n \ell$ is far from being tight for large values of $\ell$ as well. In particular, consider the symmetric case where $\ell=n$. It turns out that $u_{n}(n)=o\left(n^{2}\right)$. To see this take (perhaps the most natural choice of) arithmetic progressions: $A_{j}=\{i j \mid i \in[n]\}$ for

\footnotetext{
* Mathematics Dept., The Open University of Israel, Raanana 43107 , Israel. tipshoni@gmail.com.

${ }^{\dagger}$ Mathematics Dept., Technion-Israel Institute of Technology, Haifa 32000, Israel. room@math.technion.ac.il. Supported by ISF grant (grant No. 1357/12) and by BSF grant (grant No. 2008290).
} 
$j=1, \ldots, n$. Clearly, the union $\bigcup_{j=1}^{n} A_{j}$ is precisely the set $\{i j \mid i, j \in[n]\}$. It was shown already by Erdős in $1955([4])$ that

$$
|\{i j \mid i, j \in[n]\}|=o\left(n^{2} /(\log n)^{\alpha}\right)
$$

for some $\alpha>0$. The exact assymptotics

$$
|\{i j \mid i, j \in[n]\}| \sim \frac{n^{2}}{(\log n)^{1-\frac{1+\log \log 2}{\log 2}(\log \log n)^{\frac{3}{2}}}}
$$

was given in 2008 by Ford ([5]). Consequently, we obtain the desired improved upper bound for $u_{n}(n)$.

In this paper we show that $u_{\ell}(n)$ cannot be much smaller than $n \ell$, provided $\ell$ is not much smaller than $n$, as captured in the following theorem, giving a lower bound for $u_{\ell}(n)$ for smaller values of $\ell$ as well.

Theorem 1.1. For every $\varepsilon>0$ there is a positive constant $c_{1}(\varepsilon)$, depending only on $\varepsilon$, such that for any positive integers $n$ and $\ell$

$$
u_{\ell}(n) \geq \begin{cases}c_{1}(\varepsilon) n^{\frac{1}{2}-\varepsilon} \ell & \text { for } \ell \leq n^{\frac{1}{2}-\varepsilon} \\ c_{1}(\varepsilon) \ell^{2} & \text { for } n^{\frac{1}{2}-\varepsilon} \leq \ell \leq n^{1-\varepsilon} \\ c_{1}(\varepsilon) n^{1-\varepsilon} \ell & \text { for } n^{1-\varepsilon} \leq \ell .\end{cases}
$$

In the proof of Theorem 1.1 we study and use upper bounds for the following two functions, that are of independent interest.

$$
\begin{gathered}
f_{d}(m, n)=\max _{\substack{A, B \subset(0, \infty) \\
|A| \leq m,|B| \leq n}}\left|\left\{(a, b) \in A \times B \mid \frac{a}{b} \in[d]\right\}\right|, \\
g_{d}(n)=\max _{\substack{B \subset(0, \infty) \\
|B| \leq n}}\left|\left\{\left(b_{1}, b_{2}\right) \in B^{2} \mid b_{1}<b_{2}, \exists p, q \in[d]: \frac{b_{1}}{b_{2}}=\frac{p}{q}\right\}\right| .
\end{gathered}
$$

The paper is organized as follows. In Sections 2 we provide an upper bound for the function $g_{d}$ above. Using this bound, we provide an upper bound for the function $f_{d}$ in Section 3. Theorem 1.1 is proved in Section 4. Section 5 contains one of many possible number theory applications to the upper bounds for $f_{d}$ and $g_{d}$.

\section{Rational quotients with bounded numerator and denominator}

For positive integer $d$ define

$$
R_{d}=\left\{\frac{k}{\ell} \mid k, \ell \in[d]\right\} .
$$


Definition 2.1. For a positive integer $d$ and a finite set $B$ of positive real numbers define

$$
\mathcal{G}_{d}(B)=\left\{\left\{b_{1}, b_{2}\right\} \subseteq B \mid 1 \neq \frac{b_{1}}{b_{2}} \in R_{d}\right\} .
$$

For positive integers $n$ and $d$ define

$$
g_{d}(n)=\max _{\substack{B \subset(0, \infty) \\|B| \leq n}}\left|\mathcal{G}_{d}(B)\right|
$$

Clearly,

$$
g_{d}(n) \leq(n-1)\left|R_{d}\right|<n d^{2} .
$$

This bound is useful when $d$ is small. For large values of $d$ we have the following improved upper bound:

Proposition 2.2. For any positive integer $k$, there is a positive constant $c_{2}(k)$, depending only on $k$, such that for any positive integer $n$ and any integer $d>c_{2}(k)$

$$
g_{d}(n)<(200 k+1) n^{1+\frac{1}{k}} d^{1-\frac{1}{2 k}} .
$$

The proof of Proposition 2.2 will follow by comparing upper and lower bounds for the cardinality of the set

$$
\mathcal{C}_{d, 2 k}(B)=\left\{\left(b_{1}, b_{2}, \ldots, b_{2 k}\right) \in B^{2 k} \mid \frac{b_{1}}{b_{2}}, \frac{b_{2}}{b_{3}}, \ldots, \frac{b_{2 k-1}}{b_{2 k}}, \frac{b_{2 k}}{b_{1}} \in R_{d}, \forall 1 \leq i<j \leq 2 k: b_{i} \neq b_{j}\right\},
$$

in terms of $\left|\mathcal{G}_{d}(B)\right|$, where $B$ is a finite set of positive real numbers.

We start with bounding the cardinality of $\mathcal{C}_{d, 2 k}(B)$ from below. We first get a basic lower bound for $\left|\mathcal{C}_{d, 2 k}(B)\right|$ using the Bondy-Simonovits Theorem ([2]), which states that a graph with $n$ vertices and no simple cycles of length $2 k$ has no more than $100 k \cdot n^{1+\frac{1}{k}}$ edges. Later, we enhance this basic lower bound for $\left|\mathcal{C}_{d, 2 k}(B)\right|$ in the case where $\left|\mathcal{G}_{d}(B)\right|$ is large.

Lemma 2.3. For any positive integers $k$ and $d$, and for any finite set $B$ of positive real numbers,

$$
\frac{1}{4 k}\left|\mathcal{C}_{d, 2 k}(B)\right| \geq\left|\mathcal{G}_{d}(B)\right|-100 k|B|^{1+\frac{1}{k}}
$$

Proof. Form a graph on the vertex set $B$, by connecting two distinct vertices $b_{1}, b_{2} \in B$ if and only if $\frac{b_{1}}{b_{2}} \in R_{d}$. This graph obviously has $|B|$ vertices, $\left|\mathcal{G}_{d}(B)\right|$ edges, and at most $\frac{1}{2 \cdot 2 k}\left|\mathcal{C}_{d, 2 k}(B)\right|$ simple cycles of length $2 k$. Now remove an edge from every simple cycle of length $2 k$ in this graph. We get a graph with $|B|$ vertices and at least

$$
\left|\mathcal{G}_{d}(B)\right|-\frac{1}{4 k}\left|\mathcal{C}_{d, 2 k}(B)\right|
$$

edges. The resulting graph has no simple cycle of length $2 k$. The result now follows directly from the Bondy-Simonovits Theorem stated above. 
Next, we use a standard probabilistic argument to enhance the lower bound of Lemma 2.3 .

Lemma 2.4. For any positive integers $k$ and $d$, and for any finite set $B$ of positive real numbers such that $|B|>d^{1+\frac{1}{2(k-1)}}$, we have the following inequality:

$$
\left|\mathcal{G}_{d}(B)\right|<\frac{1}{4 k}\left|\mathcal{C}_{d, 2 k}(B)\right| d^{-(2 k-1)}+200 k n^{1+\frac{1}{k}} d^{1-\frac{1}{2 k}}
$$

Proof. Let $p:=d^{-1-\frac{1}{2(k-1)}}$, and let $B_{p}$ be a random subset of $B$ obtained by choosing each element independently with probability $p$. By Lemma 2.3,

$$
\frac{1}{4 k}\left|\mathcal{C}_{d, 2 k}\left(B_{p}\right)\right| \geq\left|\mathcal{G}_{d}\left(B_{p}\right)\right|-100 k\left|B_{p}\right|^{1+\frac{1}{k}} .
$$

Taking expectations, we get

$$
\frac{1}{4 k} E\left|\mathcal{C}_{d, 2 k}\left(B_{p}\right)\right| \geq E\left|\mathcal{G}_{d}\left(B_{p}\right)\right|-100 k E\left(\left|B_{p}\right|^{1+\frac{1}{k}}\right) .
$$

Notice that from the linearity of expectation we have:

$$
E\left|\mathcal{C}_{d, 2 k}\left(B_{p}\right)\right|=\left|\mathcal{C}_{d, 2 k}(B)\right| p^{2 k}
$$

and

$$
E\left|\mathcal{G}_{d}\left(B_{p}\right)\right|=\left|\mathcal{G}_{d}(B)\right| p^{2} .
$$

As for $E\left(\left|B_{p}\right|^{1+\frac{1}{k}}\right)$, note that $E\left|B_{p}\right|=|B| p$ and $V\left|B_{p}\right|=|B| p(1-p)$. Therefore, since $1<|B| p$,

$$
E\left(\left|B_{p}\right|^{2}\right)=V\left|B_{p}\right|+\left(E\left|B_{p}\right|\right)^{2}=|B| p(1-p)+(|B| p)^{2}<2|B|^{2} p^{2} .
$$

Now, by Holder's Inequality,

$$
E\left(\left|B_{p}\right|^{1+\frac{1}{k}}\right) \leq\left(E\left(\left|B_{p}\right|^{2}\right)\right)^{\frac{1}{2}+\frac{1}{2 k}}<2|B|^{1+\frac{1}{k}} p^{1+\frac{1}{k}} .
$$

Plugging (4), (15) and (6) in (3) we get

$$
\frac{1}{4 k}\left|\mathcal{C}_{d, 2 k}(B)\right| p^{2 k}>\left|\mathcal{G}_{d}(B)\right| p^{2}-200 k|B|^{1+\frac{1}{k}} p^{1+\frac{1}{k}},
$$

hence

$$
\left|\mathcal{G}_{d}(B)\right|<\frac{1}{4 k}\left|\mathcal{C}_{d, 2 k}(B)\right| p^{2 k-2}+200 k|B|^{1+\frac{1}{k}} p^{-1+\frac{1}{k}}=\frac{1}{4 k}\left|\mathcal{C}_{d, 2 k}(B)\right| d^{-(2 k-1)}+200 k n^{1+\frac{1}{k}} d^{1-\frac{1}{2 k}} .
$$

We now approach the task of bounding $\left|\mathcal{C}_{d, 2 k}(B)\right|$ from above. We start with the following well known number-theoretic bound on the number of divisors $d(m)$ of an integer $m$.

Lemma 2.5. For any $\delta>0$ there is a positive constant $c_{3}(\delta)$ depending only on $\delta$, such that for any positive integer $m$,

$$
d(m)<c_{3}(\delta) m^{\delta}
$$


Proof. Let $m=\prod_{i=1}^{k} p_{i}^{r_{i}}$ be the prime factorization of $m . m$ has $d(m)=\prod_{i=1}^{k}\left(1+r_{i}\right)$ divisors. For any $1 \leq i \leq k$,

$$
\left(p_{i}^{r_{i}}\right)^{\delta}=e^{\delta r_{i} \ln p_{i}}>1+\delta r_{i} \ln p_{i}
$$

Therefore,

$$
\frac{d(m)}{m^{\delta}}=\prod_{i=1}^{k} \frac{1+r_{i}}{\left(p_{i}^{r_{i}}\right)^{\delta}}<\prod_{i=1}^{k} \frac{1+r_{i}}{1+\delta r_{i} \ln p_{i}} \leq \prod_{i=1}^{k} \frac{1}{\min \left\{1, \delta \ln p_{i}\right\}}=\prod_{\substack{1 \leq i \leq k \\ \ln p_{i} \leq 1 / \delta}} \frac{1}{\delta \ln p_{i}} \leq \prod_{\substack{p \text { prime } \\ p \leq e^{1 / \delta}}} \frac{1}{\delta \ln p} .
$$

Hence, $d(m)<c_{3}(\delta) m^{\delta}$, where $c_{3}(\delta):=\prod_{\substack{p \\ p \leq e^{1 / \delta}}} \frac{1}{\delta \ln p}$.

Lemma 2.6. For any positive integer $k$ there is a positive constant $c_{4}(k)$, depending only on $k$, such that for any positive integer $d$ and any finite set $B$ of positive real numbers we have

$$
\left|\mathcal{C}_{d, 2 k}(B)\right|<c_{4}(k)|B| d^{2 k+\frac{1}{4 k}}
$$

Proof. We notice that

$$
\begin{aligned}
\left|\mathcal{C}_{d, 2 k}(B)\right| & \leq\left|\left\{\left(b_{1}, b_{2}, \ldots, b_{2 k}\right) \in B^{2 k} \mid \forall 1 \leq i \leq 2 k-1: \frac{b_{i}}{b_{i+1}} \in R_{d}, \frac{b_{2 k}}{b_{1}} \in R_{d}\right\}\right| \leq \\
& \leq|B| \cdot\left|\left\{\left(r_{1}, r_{2}, \ldots, r_{2 k}\right) \mid \forall 1 \leq i \leq 2 k: r_{i} \in R_{d}, r_{1} r_{2} \cdots r_{2 k}=1\right\}\right| \leq \\
& \leq|B| \cdot\left|\left\{\left(\left(p_{1}, p_{2}, \ldots, p_{2 k}\right),\left(q_{1}, q_{2}, \ldots, q_{2 k}\right)\right) \in\left([d]^{2 k}\right)^{2} \mid p_{1} p_{2} \cdots p_{2 k}=q_{1} q_{2} \cdots q_{2 k}\right\}\right| \leq \\
& \leq|B| \cdot \sum_{m=1}^{d^{2 k}}\left|\left\{\left(p_{1}, p_{2}, \ldots, p_{2 k}\right) \in[d]^{2 k} \mid p_{1} p_{2} \cdots p_{2 k}=m\right\}\right|^{2} \leq|B| \cdot \sum_{m=1}^{d^{2 k}} d(m)^{2 k} .
\end{aligned}
$$

By Lemma 2.5, $d(m)<c_{3}\left(1 / 16 k^{3}\right) m^{1 / 16 k^{3}}$ for any $m$, and we get

$$
\left|\mathcal{C}_{d, 2 k}(B)\right|<|B| \cdot \sum_{m=1}^{d^{2 k}}\left(c_{3}\left(1 / 16 k^{3}\right) m^{1 / 16 k^{3}}\right)^{2 k} \leq\left(c_{3}\left(1 / 16 k^{3}\right)\right)^{2 k}|B| d^{2 k+\frac{1}{4 k}}
$$

This completes the proof with $c_{4}(k):=\left(c_{3}\left(1 / 16 k^{3}\right)\right)^{2 k}$.

We are now prepared for proving Proposition 2.2 .

Proof of Proposition 2.2. If $n \leq d^{1+\frac{1}{2(k-1)}}$, then (2) holds because

$$
g_{d}(n) \leq\left(\begin{array}{l}
n \\
2
\end{array}\right)<n^{2}=n^{1+\frac{1}{k}} n^{1-\frac{1}{k}} \leq n^{1+\frac{1}{k}}\left(d^{1+\frac{1}{2(k-1)}}\right)^{1-\frac{1}{k}}=n^{1+\frac{1}{k}} d^{1-\frac{1}{2 k}}
$$

We therefore assume $n>d^{1+\frac{1}{2(k-1)}}$. Let $B$ be a set of $n$ positive real numbers. By Lemma 2.4.

$$
\left|\mathcal{G}_{d}(B)\right|<200 k n^{1+\frac{1}{k}} d^{1-\frac{1}{2 k}}+\frac{1}{4 k}\left|\mathcal{C}_{d, 2 k}(B)\right| d^{-(2 k-1)} .
$$


By Lemma 2.6,

$$
\left|\mathcal{C}_{d, 2 k}(B)\right|<c_{4}(k) n d^{2 k+\frac{1}{4 k}} .
$$

Hence, for $d \geq c_{2}(k):=\left(c_{4}(k) / 4 k\right)^{4 k}$,

$$
\left|\mathcal{C}_{d, 2 k}(B)\right|<4 k n d^{2 k+\frac{1}{2 k}} .
$$

Plugging (8) in (7) and using our assumption that $n>d^{1+\frac{1}{2(k-1)}} \geq d$, we get that for $d \geq c_{2}(k)$,

$$
\left|\mathcal{G}_{d}(B)\right|<200 k n^{1+\frac{1}{k}} d^{1-\frac{1}{2 k}}+n d^{1+\frac{1}{2 k}}<(200 k+1) n^{1+\frac{1}{k}} d^{1-\frac{1}{2 k}} .
$$

\section{Bounded integer quotients}

Definition 3.1. For positive integers $m, n$, and $d$ define

$$
f_{d}(m, n)=\max _{\substack{A, B \subset(0, \infty) \\|A| \leq m,|B| \leq n}}\left|\left\{(a, b) \in A \times B \mid \frac{a}{b} \in[d]\right\}\right| .
$$

Remark 3.2. It is an amusing exercise to see that $f_{d}(m, n)=f_{d}(n, m)$. Therefore, we may assume, if needed, with no loss of generality that $m \leq n$, or that $m \geq n$.

Proposition 3.3. For any $\varepsilon>0$ there is a positive constant $c_{6}(\varepsilon)$, depending only on $\varepsilon$, such that for any positive integers $m, n$, and $d$

$$
f_{d}(m, n)<c_{5}(\varepsilon)(\min \{\sqrt{n}, \sqrt{m}\})^{\varepsilon} \sqrt{m n d} .
$$

Proof. With no loss of generality (see Remark 3.2) assume that $n \leq m$. We may also assume that $m<n d$, because if $m \geq n d$, then $f_{d}(m, n) \leq n d=\sqrt{(n d) n d} \leq \sqrt{m n d}$ (see Proposition 3.5 for further discussion).

Let $A$ and $B$ be finite sets of positive real numbers such that $|A| \leq m,|B| \leq n$. The proposition will follow by comparing lower and upper bounds for the cardinality of the set

$$
W=\left\{\left(a, b_{1}, b_{2}\right) \in A \times B^{2} \mid \frac{a}{b_{1}}, \frac{a}{b_{2}} \in[d], \quad b_{1}<b_{2}\right\} .
$$

We first establish an upper bound for $|W|$. For convenience define

$$
S_{d}=\{(p, q) \mid p, q \in[d], \quad p<q, \quad \operatorname{gcd}(p, q)=1\} .
$$

We have: 


$$
\begin{aligned}
|W| & =\left|\left\{\left(a, b_{1}, b_{2}\right) \in A \times B^{2} \mid \frac{a}{b_{1}}, \frac{a}{b_{2}} \in[d], \quad b_{1}<b_{2}\right\}\right|= \\
& =\left|\left\{\left(b_{1}, b_{2}, k_{1}, k_{2}\right) \in B^{2} \times[d]^{2} \mid k_{1} b_{1}=k_{2} b_{2} \in A, \quad b_{1}<b_{2}\right\}\right| \leq \\
& \leq\left|\left\{\left(b_{1}, b_{2}, k_{1}, k_{2}\right) \in B^{2} \times[d]^{2} \mid k_{1} b_{1}=k_{2} b_{2}, \quad b_{1}<b_{2}\right\}\right|= \\
& =\sum_{(p, q) \in S_{d}}\left|\left\{\left(b_{1}, b_{2}, k_{1}, k_{2}\right) \in B^{2} \times[d]^{2} \mid \frac{b_{1}}{b_{2}}=\frac{k_{2}}{k_{1}}=\frac{p}{q}\right\}\right|= \\
& =\sum_{(p, q) \in S_{d}}\left|\left\{\left(b_{1}, b_{2}\right) \in B^{2} \mid \frac{b_{1}}{b_{2}}=\frac{p}{q}\right\}\right| \cdot\left|\left\{\left(k_{1}, k_{2}\right) \in[d]^{2} \mid \frac{k_{2}}{k_{1}}=\frac{p}{q}\right\}\right|= \\
& =\sum_{(p, q) \in S_{d}}\left|\left\{\left(b_{1}, b_{2}\right) \in B^{2} \mid \frac{b_{1}}{b_{2}}=\frac{p}{q}\right\}\right| \cdot\left\lfloor\frac{d}{q}\right\rfloor=\sum_{q=2}^{d}\left(\left|\mathcal{G}_{q}(B)\right|-\left|\mathcal{G}_{q-1}(B)\right|\right)\left\lfloor\frac{d}{q}\right\rfloor \leq \\
\leq & \sum_{q=2}^{d}\left(\left|\mathcal{G}_{q}(B)\right|-\left|\mathcal{G}_{q-1}(B)\right|\right) \frac{d}{q}=\left|\mathcal{G}_{d}(B)\right|+\sum_{q=2}^{d-1}\left|\mathcal{G}_{q}(B)\right|\left(\frac{d}{q}-\frac{d}{q+1}\right)= \\
& =\left|\mathcal{G}_{d}(B)\right|+\sum_{q=2}^{d-1}\left|\mathcal{G}_{q}(B)\right| \frac{d}{q(q+1)} .
\end{aligned}
$$

Let $k:=\max \{\lceil 1 / \varepsilon\rceil, 1\}$. By Proposition 2.2 , there is a positive constant $c_{2}(k)$, depending only on $k$, such that for any $c_{2}(k)<q \leq d$,

$$
\left|\mathcal{G}_{q}(B)\right|<(200 k+1) n^{1+\frac{1}{k}} q^{1-\frac{1}{2 k}} .
$$

For any $q$, we have by (11) that $\left|\mathcal{G}_{q}(B)\right|<n q^{2}$.

Therefore,

$$
\begin{aligned}
|W| & \leq\left|\mathcal{G}_{d}(B)\right|+\sum_{q=2}^{c_{2}(k)}\left|\mathcal{G}_{q}(B)\right| \frac{d}{q(q+1)}+\sum_{q=c_{2}(k)+1}^{d-1}\left|\mathcal{G}_{q}(B)\right| \frac{d}{q(q+1)}< \\
& <(200 k+1) n^{1+\frac{1}{k}} d^{1-\frac{1}{2 k}}+\sum_{q=2}^{c_{2}(k)} n q^{2} \frac{d}{q(q+1)}+\sum_{q=c_{2}(k)+1}^{d-1}(200 k+1) n^{1+\frac{1}{k}} q^{1-\frac{1}{2 k}} \frac{d}{q(q+1)} \leq \\
& \leq(200 k+1) n^{1+\varepsilon} d^{1-\frac{1}{2 k}}+\left(c_{2}(k)-1\right) n d+(200 k+1) n^{1+\varepsilon} d \sum_{q=c_{2}(k)+1}^{d-1} \frac{1}{q^{1+\frac{1}{2 k}}} .
\end{aligned}
$$

Hence,

$$
|W|<c(\varepsilon) n^{1+\varepsilon} d,
$$

where $c(\varepsilon):=(200 k+1)+\left(c_{2}(k)-1\right)+(200 k+1) \sum_{q=c_{2}(k)+1}^{\infty} \frac{1}{q^{1+\frac{1}{2 k}}}$. 
To get a lower bound for $|W|$, we define $d(a)=\left|\left\{b \in B \mid \frac{a}{b} \in[d]\right\}\right|$ for any $a \in A$. Then, by the convexity of the function $\left(\begin{array}{l}x \\ 2\end{array}\right)=\frac{x(x-1)}{2}$ :

$$
|W|=\sum_{a \in A}\left(\begin{array}{c}
d(a) \\
2
\end{array}\right) \geq m\left(\begin{array}{c}
\frac{1}{m} \sum_{a \in A} d(a) \\
2
\end{array}\right) .
$$

Combining the upper and lower bounds for $|W|$, namely, (9) and (10), we get

$$
m\left(\begin{array}{c}
\frac{1}{m} \sum_{a \in A} d(a) \\
2
\end{array}\right)<c(\varepsilon) n^{1+\varepsilon} d .
$$

Now, we deduce

$$
\left|\left\{(a, b) \in A \times B \mid \frac{a}{b} \in[d]\right\}\right|=\sum_{a \in A} d(a)<\frac{m}{2}+\sqrt{\frac{m^{2}}{4}+2 c(\varepsilon) m n^{1+\varepsilon} d} .
$$

This implies the desired result, as $n \leq m<n d$.

\subsection{Tightness of Proposition 3.3}

In this section we will show that the upper bound in Proposition 3.3 for $f_{d}(n, m)$ is essentially tight (see Proposition 3.4 below), provided that none of the parameters $m, n$, and $d$ is much larger than the product of the other two. When one of $m, n$, and $d$ is considerably larger than the product of the other two, the upper bound in Proposition 3.3 is no longer tight, as follows from Proposition 3.5 below, in which the exact values of $f_{d}(m, n)$ in those cases are determined.

Proposition 3.4. If $m \leq 4 n d, n \leq 4 m d$, and $d \leq 4 m n$, then $f_{d}(m, n) \geq \frac{1}{8} \sqrt{m n d}$.

Proof. Set $k=\lfloor\sqrt{m d / n}\rfloor, \ell=\lfloor\sqrt{n d / m}\rfloor$, and $t=\lfloor\sqrt{m n / d}\rfloor$. Consider the sets

$$
A=\left\{(k+\ell)^{r} i\right\}_{r \in[t], i \in[k]}, \quad \text { and } \quad B=\left\{(k+\ell)^{r} / j\right\}_{r \in[t], j \in[\ell]} .
$$

Then

$$
\begin{gathered}
|A|=t k \leq \sqrt{\frac{m n}{d}} \cdot \sqrt{\frac{m d}{n}}=m \text { and } \\
|B|=t \ell \leq \sqrt{\frac{m n}{d}} \cdot \sqrt{\frac{n d}{m}}=n .
\end{gathered}
$$

Notice that

$$
\left|\left\{(a, b) \in A \times B \mid \frac{a}{b} \in[d]\right\}\right| \geq t k \ell \geq \frac{1}{2} \sqrt{\frac{m n}{d}} \cdot \frac{1}{2} \sqrt{\frac{m d}{n}} \cdot \frac{1}{2} \sqrt{\frac{n d}{m}}=\frac{1}{8} \sqrt{m n d} .
$$

Proposition 3.5. 1. If $d \geq m n$ then $f_{d}(m, n)=m n$. 
2. If $n \geq m d$ then $f_{d}(m, n)=m d$.

3. If $m \geq n d$ then $f_{d}(m, n)=n d$.

Proof. $\quad$ 1. For any $A, B \subset(0, \infty)$ with $|A| \leq m,|B| \leq n$ we obviously have

$$
\left|\left\{(a, b) \in A \times B \mid \frac{a}{b} \in[d]\right\}\right| \leq|A \times B|=|A| \cdot|B| \leq m n .
$$

To see that this upper bound can actually be attained, consider, for instance, the sets $A=$ $\{1 / i\}_{i \in[m]}$ and $B=[n]$.

2. For any $A, B \subset(0, \infty)$ with $|A| \leq m,|B| \leq n$ we have

$$
\left|\left\{(a, b) \in A \times B \mid \frac{a}{b} \in[d]\right\}\right|=\left|\left\{(a, k) \in A \times[d] \mid \frac{a}{k} \in B\right\}\right| \leq m d .
$$

This upper bound can indeed be attained, for example by taking $A=\left\{(d+1)^{i}\right\}_{i \in[m]}$ and $B=\left\{(d+1)^{i} / k\right\}_{i \in[m], k \in[d]}$.

3. For any $A, B \subset(0, \infty)$ with $|A| \leq m,|B| \leq n$ we have

$$
\left|\left\{(a, b) \in A \times B \mid \frac{a}{b} \in[d]\right\}\right|=|\{(b, k) \in B \times[d] \mid k \cdot b \in A\}| \leq n d .
$$

Equality is attained, for example, by taking $A=\left\{(d+1)^{j} k\right\}_{j \in[n], k \in[d]}$ and $B=\left\{(d+1)^{j}\right\}_{j \in[n]}$.

\section{Union of arithmetic progressions}

In this Section we prove Theorem 1.1.

Recall that for integers $n>1$ and $\ell>1, u_{\ell}(n)$ is the minimum possible cardinality of a union of $n$ arithmetic progressions, each of length $\ell$, with pairwise distinct differences.

As a consequence of Proposition 3.3 we get the following easy lower bound for $u_{\ell}(n)$ that will be useful in the regime $\ell \leq n^{\frac{1}{2}-\varepsilon}$ :

Proposition 4.1. For any $\varepsilon>0$ there is a positive constant $c_{6}(\varepsilon)$, depending only on $\varepsilon$, such that for any positive integers $n$ and $\ell$

$$
u_{\ell}(n)>c_{6}(\varepsilon) n^{\frac{1}{2}-\varepsilon} \ell
$$

Proof. Take $n$ arithmetic progressions, each of length $\ell$, with pairwise distinct differences, and let $U$ be their union. If each $x \in U$ belongs to less than $\sqrt{n}$ of the progressions, then $n \ell<|U| \sqrt{n}$ and consequently $|U|>\sqrt{n} \ell>n^{\frac{1}{2}-\varepsilon} \ell$.

Therefore, assume there is $x \in U$ which belongs to at least $\sqrt{n}$ progressions. In any such progression at least $d:=\left\lceil\frac{\ell-1}{2}\right\rceil$ of the terms are on the same side of $x$ (that is, either come before or after). Therefore, in at least $\sqrt{n} / 2$ progressions there are at least $d$ terms on the same side of $x$ and 
without loss of generality we assume they come after $x$ in these progressions. We now concentrate only on these progressions. Let $B$ be the set of differences of these arithmetic progressions, and let $A=\{i b \mid i \in[d], \quad b \in B\}$. Proposition 3.3 implies

$$
\begin{aligned}
d|B| & =\left|\left\{(a, b) \in A \times B \mid \frac{a}{b} \in[d]\right\}\right| \leq f_{d}(|A|,|B|)< \\
& <c_{5}(\varepsilon)(\min \{\sqrt{|A|}, \sqrt{|B|}\})^{\varepsilon} \sqrt{|A||B| d} \leq c_{5}(\varepsilon) \sqrt{|B|^{\varepsilon}} \cdot \sqrt{|A||B| d}
\end{aligned}
$$

hence

$$
|U| \geq|\{x+a \mid a \in A\}|=|A|>\frac{1}{c_{5}(\varepsilon)^{2}}|B|^{1-\varepsilon} d \geq \frac{1}{c_{5}(\varepsilon)^{2}}\left(\frac{\sqrt{n}}{2}\right)^{1-\varepsilon} \frac{\ell-1}{2} \geq \frac{1}{c_{5}(\varepsilon)^{2} 2^{3-\varepsilon}} n^{\frac{1}{2}-\varepsilon} \ell .
$$

This completes the proof with $c_{6}(\varepsilon):=\min \left\{1, \frac{1}{c_{5}(\varepsilon)^{2} 2^{3-\varepsilon}}\right\}$.

The lower bounds in Theorem 1.1 in the regime $n^{\frac{1}{2}-\varepsilon} \leq \ell$ are established in Proposition 4.3 below. The proof of Proposition 4.3 uses Proposition 2.2, ideas similar to those appearing in the proof of Proposition 3.3, and the following lemma (recall the definition of $R_{d}$ from Section 2).

Lemma 4.2. Suppose that the arithmetic progressions $\left(a_{1}+(j-1) b_{1}\right)_{j=1}^{\ell}$ and $\left(a_{2}+(j-1) b_{2}\right)_{j=1}^{\ell}$ have at least $r \geq 2$ common elements, then

$$
\frac{b_{1}}{b_{2}} \in R_{\left\lfloor\frac{\ell-1}{r-1}\right\rfloor}
$$

Proof. The intersection of the arithmetic progressions $\left(a_{1}+(j-1) b_{1}\right)_{j=1}^{\ell}$ and $\left(a_{2}+(j-1) b_{2}\right)_{j=1}^{\ell}$ is in itself an arithmetic progression, whose length is at least $r \geq 2$. Suppose $a_{1}+\left(i_{1}-1\right) b_{1}=$ $a_{2}+\left(i_{2}-1\right) b_{2}$ and $a_{1}+\left(j_{1}-1\right) b_{1}=a_{2}+\left(j_{2}-1\right) b_{2}$ are, respectively, the first and second terms of this arithmetic progression. Since the progression is of length at least $r$, then $(r-1)\left(j_{1}-i_{1}\right) \leq \ell-1$ and $(r-1)\left(j_{2}-i_{2}\right) \leq \ell-1$. It follows that $j_{1}-i_{1} \leq\left\lfloor\frac{\ell-1}{r-1}\right\rfloor$ and $j_{2}-i_{2} \leq\left\lfloor\frac{\ell-1}{r-1}\right\rfloor$.

We also have

$\left(j_{1}-i_{1}\right) b_{1}=\left(a_{1}+\left(j_{1}-1\right) b_{1}\right)-\left(a_{1}+\left(i_{1}-1\right) b_{1}\right)=\left(a_{2}+\left(j_{2}-1\right) b_{2}\right)-\left(a_{2}+\left(i_{2}-1\right) b_{2}\right)=\left(j_{2}-i_{2}\right) b_{2}$.

Consequently,

$$
\frac{b_{1}}{b_{2}}=\frac{j_{2}-i_{2}}{j_{1}-i_{1}} \in R_{\left\lfloor\frac{\ell-1}{r-1}\right\rfloor}
$$

Proposition 4.3. For any $\varepsilon>0$ there is a positive constant $c_{7}(\varepsilon)$, depending only on $\varepsilon$, such that for any positive integers $n$ and $\ell$

$$
u_{\ell}(n)>c_{7}(\varepsilon) \min \left\{n^{1-\varepsilon} \ell, \ell^{2}\right\}
$$


Proof. Let $P_{1}, P_{2}, \ldots, P_{n}$ be $n$ arithmetic progressions, each of length $\ell$, with pairwise distinct differences. We write $P_{i}=\left\{a_{i}+(j-1) b_{i}\right\}_{j \in[\ell]}$ for $i \in[n]$, and let $U=\bigcup_{i=1}^{n} P_{i}=\left\{a_{i}+(j-1) b_{i} \mid i \in[n], j \in\right.$ $[\ell]\}$ be the union of these arithmetic progressions. For every $x \in U$ let $\alpha(x)=\left|\left\{i \in[n] \mid x \in P_{i}\right\}\right|$ be the number of progressions containing $x$. Clearly, $\sum_{x \in U} \alpha(x)=n \ell$. The proof will follow by comparing lower and upper bounds for the cardinality of the set

$$
W=\left\{\left(x, i_{1}, i_{2}\right) \in U \times[n]^{2} \mid x \in P_{i_{1}} \cap P_{i_{2}}, \quad i_{1}<i_{2}\right\} .
$$

To get an upper bound on $|W|$ notice that

$$
|W|=\sum_{1 \leq i_{1}<i_{2} \leq n}\left|P_{i_{1}} \cap P_{i_{2}}\right|=\sum_{r=1}^{\ell-1}\left|\left\{\left(i_{1}, i_{2}\right) \in[n]^{2}\left|b_{i_{1}}<b_{i_{2}}, \quad\right| P_{i_{1}} \cap P_{i_{2}} \mid \geq r\right\}\right| .
$$

Trivially,

$$
\left|\left\{\left(i_{1}, i_{2}\right) \in[n]^{2}\left|b_{i_{1}}<b_{i_{2}}, \quad\right| P_{i_{1}} \cap P_{i_{2}} \mid \geq 1\right\}\right| \leq\left(\begin{array}{c}
n \\
2
\end{array}\right) .
$$

For $r \geq 2$ we use Lemma 4.2 to obtain

$$
\left|\left\{\left(i_{1}, i_{2}\right) \in[n]^{2}\left|b_{i_{1}}<b_{i_{2}}, \quad\right| P_{i_{1}} \cap P_{i_{2}} \mid \geq r\right\}\right| \leq\left|\mathcal{G}_{\left\lfloor\frac{\ell-1}{r-1}\right\rfloor}(B)\right| \leq g_{\left\lfloor\frac{\ell-1}{r-1}\right\rfloor}(n) .
$$

Hence,

$$
|W| \leq\left(\begin{array}{l}
n \\
2
\end{array}\right)+\sum_{r=2}^{\ell-1} g_{\left\lfloor\frac{\ell-1}{r-1}\right\rfloor}(n) .
$$

Let $k:=\max \{\lceil 1 / \varepsilon\rceil, 1\}$. By Proposition 2.2 , there is a constant $c_{2}(k)$ such that for any $2 \leq r \leq$ $\left\lfloor\frac{\ell-1}{c_{2}(k)+1}\right\rfloor+1$ we have

$$
g_{\left\lfloor\frac{\ell-1}{r-1}\right\rfloor}(n)<(200 k+1) n^{1+\frac{1}{k}}\left(\left\lfloor\frac{\ell-1}{r-1}\right\rfloor\right)^{1-\frac{1}{2 k}} \leq(200 k+1) n^{1+\frac{1}{k}}(\ell-1)^{1-\frac{1}{2 k}} \frac{1}{r^{1-\frac{1}{2 k}}} .
$$

For $\left\lfloor\frac{\ell-1}{c_{2}(k)+1}\right\rfloor+2 \leq r \leq \ell-1$, we use the simpler estimate (11) to get

$$
g_{\left\lfloor\frac{\ell-1}{r-1}\right\rfloor}(n)<n\left(\left\lfloor\frac{\ell-1}{r-1}\right\rfloor\right)^{2}<\left(c_{2}(k)+1\right)^{2} n .
$$

Therefore,

$$
|W| \leq\left(\begin{array}{l}
n \\
2
\end{array}\right)+\sum_{r=2}^{\left\lfloor\frac{\ell-1}{c_{2}(k)+1}\right\rfloor+1}(200 k+1) n^{1+\frac{1}{k}}(\ell-1)^{1-\frac{1}{2 k}} \frac{1}{r^{1-\frac{1}{2 k}}}+\sum_{r=\left\lfloor\frac{\ell-1}{c_{2}(k)+1}\right\rfloor+2}^{\ell-1}\left(c_{2}(k)+1\right)^{2} n
$$

Hence

$$
|W|<c(\varepsilon) n \ell \max \left\{n / \ell, n^{1 / k}\right\} \mid \leq c(\varepsilon) n \ell \max \left\{n / \ell, n^{\varepsilon}\right\},
$$

for some positive constant $c(\varepsilon)$ depending only on $\varepsilon$. 
A simple lower bound for $|W|$ follows from the convexity of $\left(\begin{array}{l}x \\ 2\end{array}\right)=\frac{x(x-1)}{2}$ :

$$
|W|=\sum_{x \in U}\left(\begin{array}{c}
\alpha(x) \\
2
\end{array}\right) \geq|U|\left(\begin{array}{c}
\frac{1}{|U|} \sum_{x \in U} \alpha(x) \\
2
\end{array}\right)=|U|\left(\begin{array}{c}
n \ell /|U| \\
2
\end{array}\right) .
$$

Comparing the upper and lower bounds for $|W|$, namely, (11) and (12), we get

$$
|U|\left(\begin{array}{c}
n \ell /|U| \\
2
\end{array}\right)<c(\varepsilon) n \ell \max \left\{n / \ell, n^{\varepsilon}\right\} \text {. }
$$

Hence

$$
|U|>\frac{n \ell}{1+2 c(\varepsilon) \max \left\{n / \ell, n^{\varepsilon}\right\}},
$$

and the result follows.

Combining Proposition 4.1 and Proposition 4.3, we get

Theorem 1.1. For any $\varepsilon>0$ there is a positive constant $c_{1}(\varepsilon)$, depending only on $\varepsilon$, such that for any positive integers $n$ and $\ell$

$$
u_{\ell}(n) \geq \begin{cases}c_{1}(\varepsilon) n^{\frac{1}{2}-\varepsilon} \ell & \text { for } \ell \leq n^{\frac{1}{2}-\varepsilon} \\ c_{1}(\varepsilon) \ell^{2} & \text { for } n^{\frac{1}{2}-\varepsilon} \leq \ell \leq n^{1-\varepsilon} \\ c_{1}(\varepsilon) n^{1-\varepsilon} \ell & \text { for } n^{1-\varepsilon} \leq \ell\end{cases}
$$

\section{$5 \quad$ Further applications}

In this section we draw one (among many) possible number theoretical application to our upper bounds for the functions $f_{d}$ and $g_{d}$ in Sections 2 and 3 ,

Theorem 5.1. For every $\varepsilon>0$ there exists $c(\varepsilon)>0$ with the following property. Let $a_{1}<\ldots<a_{n}$ be $n$ natural numbers. Then

$$
\sum_{1 \leq i<j \leq n} \frac{\operatorname{gcd}\left(a_{i}, a_{j}\right)}{a_{j}}<c(\varepsilon) n^{1+\varepsilon}
$$

Proof. Denote $B=\left\{a_{1}, \ldots, a_{n}\right\}$. Notice that every summand on the left hand side of (13) is of the form $\frac{1}{k}$ for some positive integer $k$. The simple but crucial observation is that if $1 \leq i<j \leq n$ such that $\frac{\operatorname{gcd}\left(a_{i}, a_{j}\right)}{a_{j}}=\frac{1}{k}$, then $\frac{a_{i}}{a_{j}} \in R_{k}$. Therefore, $\frac{\operatorname{gcd}\left(a_{i}, a_{j}\right)}{a_{j}}=\frac{1}{d}$, for $1 \leq i<j \leq n$, if and only if $\left\{a_{i}, a_{j}\right\} \in \mathcal{G}_{d}(B) \backslash \mathcal{G}_{d-1}(B)$. (Recall the definition of $R_{k}$ and $\mathcal{G}_{d}(B)$ in Section 2.)

Fix a positive integer $k$, to be determined later. By Proposition 2.2. there exists $c_{2}(k)>0$ such that for every $d>c_{2}(k)$

$$
\left|\mathcal{G}_{d}(B)\right| \leq g_{d}(n)<(200 k+1) n^{1+\frac{1}{k}} d^{1-\frac{1}{2 k}}
$$


For every $d,\left|\mathcal{G}_{d}(B)\right| \leq g_{d}(n)<n d^{2}$, by (1). This easy upper bound will be useful when $d$ is small (smaller than $\left.c_{2}(k)\right)$.

We are now ready to prove the Theorem.

$$
\begin{aligned}
\sum_{1 \leq i<j \leq n} \frac{\operatorname{gcd}\left(a_{i}, a_{j}\right)}{a_{j}} & =\sum_{d \geq 2}\left|\left\{(i, j) \in[n]^{2} \mid i<j, \frac{\operatorname{gcd}\left(a_{i}, a_{j}\right)}{a_{j}}=\frac{1}{d}\right\}\right| \cdot \frac{1}{d}= \\
& =\sum_{d \geq 2}\left(\left|\mathcal{G}_{d}(B)\right|-\left|\mathcal{G}_{d-1}(B)\right|\right) \frac{1}{d}=\sum_{d \geq 2}\left|\mathcal{G}_{d}(B)\right|\left(\frac{1}{d}-\frac{1}{d+1}\right)= \\
& =\sum_{2 \leq d \leq c_{2}(k)} \frac{1}{d(d+1)}\left|\mathcal{G}_{d}(B)\right|+\sum_{d>c_{2}(k)} \frac{1}{d(d+1)}\left|\mathcal{G}_{d}(B)\right|< \\
& <\sum_{2 \leq d \leq c_{2}(k)} \frac{1}{d(d+1)} n d^{2}+\sum_{d>c_{2}(k)} \frac{1}{d(d+1)}(200 k+1) n^{1+\frac{1}{k}} d^{1-\frac{1}{2 k}} \leq \\
& \leq c_{2}(k) n+(200 k+1) n^{1+\frac{1}{k}} \sum_{d>c_{2}(k)} \frac{1}{d^{1 / 2 k}(d+1)} .
\end{aligned}
$$

Take $k$ to be a positive integer such that $\frac{1}{k}<\varepsilon$ and let $c(\varepsilon)=c_{2}(k)+(200 k+1) \sum_{d>c_{2}(k)} \frac{1}{d^{1 / 2 k}(d+1)}$ to get the desired result.

Remark. It is not hard to verify that the bound in Theorem 5.1 cannot be improved to be linear in $n$. This can be seen for example by taking $a_{1}, \ldots, a_{n}$ to be $1, \ldots, n$, respectively. Then a direct computation, using some classical number theory estimates, show that in this case the left hand side of (13) is $\Theta(n \log n)$.

Theorem 5.1 allows us to write in a slightly different way the proof of Proposition 4.3, giving the lower bound for $u_{\ell}(n)$.

Indeed, suppose we wish to bound from below the union of $n$ arithmetic progressions, $A_{1}, \ldots, A_{n}$, each of length $\ell$, with pairwise distinct differences $a_{1}, \ldots, a_{n}$, respectively. With no loss of generality we may assume that $a_{1}<\ldots<a_{n}$ and that they are all positive integers. We will use the following well known estimate of Dawson and Sankoff ([3]) on the cardinality of the union of sets via the cardinalities of their pairwise intersections.

$$
\left|\bigcup_{i=1}^{n} A_{i}\right| \geq \frac{\left(\sum_{i=1}^{n}\left|A_{i}\right|\right)^{2}}{\sum_{1 \leq i, j \leq n}\left|A_{i} \cap A_{j}\right|} .
$$

Hence, we examine the cardinalities of the pairwise intersections of the progressions.

Consider two progressions of length $\ell:\{p+(j-1) q\}_{j=1}^{\ell}$ and $\left\{p^{\prime}+(j-1) q^{\prime}\right\}_{j=1}^{\ell}$, where $q, q^{\prime}$ are positive integers. Their intersection is in itself an arithmetic progression and it is not hard to see that the difference of this progression (assuming it has at least two elements) is equal to the smallest number divisible by both $q$ and $q^{\prime}$. It follows that the size of the intersection of the two progressions is less than or equal to $1+\frac{\min \left(\ell q, \ell q^{\prime}\right)}{\operatorname{lcm}\left(q, q^{\prime}\right)}=1+\ell \frac{\operatorname{gcd}\left(q, q^{\prime}\right)}{\max \left(q, q^{\prime}\right)}$. 
It follows from the above discussion that the union $\left|\bigcup_{i=1}^{n} A_{i}\right|$ is bounded from below by

$$
\frac{(n \ell)^{2}}{n \ell+n^{2}+2 \ell \sum_{1 \leq i<j \leq n} \frac{\operatorname{gcd}\left(a_{i}, a_{j}\right)}{a_{j}}} .
$$

In view of Theorem 5.1, this expression is greater than $\min \left(\frac{1}{3 c(\varepsilon)} n^{1-\varepsilon} \ell, \frac{1}{2} \ell^{2}\right)$.

It is interesting to note the relation of Theorem 5.1 to a well known conjecture of Graham ([6]). Graham conjectured that given any $n$ positive integers $a_{1}<\ldots<a_{n}$, there are two of them that satisfy $\frac{a_{j}}{\operatorname{gcd}\left(a_{i}, a_{j}\right)} \geq n$. This conjecture has a long history with many contributions. It was finally completely (that is, for all values of $n$ ) solved in [1], where one can also find more details on the history and references related to this conjecture.

From (13) it follows that there is a pair of indices $1 \leq i<j \leq n$ such that $\frac{\operatorname{gcd}\left(a_{i}, a_{j}\right)}{a_{j}}<\frac{c(\varepsilon) n^{1+\varepsilon}}{\left(\begin{array}{c}n \\ 2\end{array}\right)}$. This implies $\frac{a_{j}}{\operatorname{gcd}\left(a_{i}, a_{j}\right)}>\frac{1}{2 c(\varepsilon)} n^{1-\varepsilon}$. This lower bound is indeed much weaker than the desired one in the conjecture of Graham, but on the other hand this argument shows that "in average" $\frac{a_{j}}{\operatorname{gcd}\left(a_{i}, a_{j}\right)}$ is quite large.

\section{Acknowledgments}

We thank Seva Lev for interesting discussions about the problem and for pointing out the relation of Theorem 5.1 to the conjecture of Graham.

\section{References}

[1] R. Balasubramanian, K. Soundararajan, On a conjecture of R. L. Graham. Acta Arith. 75 (1996), no. 1, 1-38.

[2] J. A. Bondy and M. Simonovits, Cycles of even length in graphs, J. Combinatorial Theory Ser. B 16 (1974), 97-105.

[3] D. A. Dawson and D. Sankoff, An inequality for probabilities, Proc. Amer. Math. Soc. 18 (1967), $504-507$.

[4] P. Erdös, Some remarks on number theory, Riveon Lematematika 9 (1955), 45-48.

[5] K. Ford, The distribution of integers with a divisor in a given interval, Ann. of Math. (2) 168 (2008), no. 2, 367-433.

[6] R. L. Graham, Advanced Problem 5749*, Amer. Math. Monthly 77 (1970), 775.

[7] I. Z. Ruzsa, On the number of sums and differences, Acta Math. Hungar. 59 (1992), no. 3-4, 439-447. 